\title{
Genome sequencing of the vermicompost strain Stenotrophomonas maltophilia UENF-4GII and population structure analysis of the $S$. maltophilia Sm3 genogroup
}

\author{
Francisnei Pedrosa-Silva ${ }^{a}$, Filipe P. Matteoli ${ }^{a}$, Hemanoel Passarelli-Araujo ${ }^{a, b}$, Fabio L.
} Olivares $^{c, d}$, Thiago M. Venancio ${ }^{a, *}$

\begin{abstract}
a Laboratório de Química e Função de Proteínas e Peptídeos, Centro de Biociências e Biotecnologia, Universidade Estadual do Norte Fluminense Darcy Ribeiro (UENF), Brazil; b Departamento de Bioquímica e Imunologia, Instituto de Ciências Biológicas, Universidade Federal de Minas Gerais, Belo Horizonte, MG, Brazil; ' Núcleo de Desenvolvimento de Insumos Biológicos para a Agricultura (NUDIBA), UENF, Brazil; ${ }^{d}$ Laboratório de Biologia Celular e Tecidual, Centro de Biociências e Biotecnologia, UENF, Brazil.
\end{abstract}

\section{* Corresponding author:}

Thiago M. Venancio; Laboratório de Química e Função de Proteínas e Peptídeos, Centro de Biociências e Biotecnologia, Universidade Estadual do Norte Fluminense Darcy Ribeiro (UENF); Av. Alberto Lamego 2000, P5 / sala 217; Campos dos Goytacazes, Rio de Janeiro, Brazil. E-mail: thiago.venancio@gmail.com.

\section{ABSTRACT}

The Stenotrophomonas maltophilia complex (Smc) is a cosmopolitan bacterial group that has been proposed an emergent multidrug-resistant pathogen. Taxonomic studies support the genomic heterogeneity of Smc, which comprises genogroups exhibiting a range of phenotypically distinct strains from different sources. Here, we report the genome sequencing and in-depth analysis of $S$. maltophilia UENF-4GII, isolated from vermicompost. This genome harbors a unique region encoding a penicillin-binding protein $(p b p \mathrm{X})$ that was carried by a transposon, as well as horizontally-transferred genomic islands involved in anti-phage defense via DNA modification, and pili glycosylation. We also analyzed all available Smc genomes to investigate genes associated with resistance and virulence, niche occupation, and population structure. $S$. maltophilia UENF-4GII belongs to genogroup 3 (Sm3), which comprises three phylogenetic clusters (PC). Pan-GWAS analysis uncovered 471 environment-associated and 791 PC-associated genes, including antimicrobial resistance (e.g. blaL1 and blaR1) and virulence determinants (e.g. treS and $k a t G$ ) that provide insights on the resistance and virulence potential of Sm3 strains. Together, the results presented here provide the grounds for more detailed clinical and ecological investigations of S. maltophilia. 


\section{INTRODUCTION}

Vermicomposting is a non-thermophilic biodegradation technique used to manage organic waste [1]. The process involves synergistic interactions between earthworms and microorganisms to biodegrade different organic waste into a humus-like material known as vermicompost, a nutrient-rich organic amendment used to enhance soil microbial diversity and plant development $[2,3]$. Vermicomposts harbor soil bacteria from various genera, such as Bacillus, Pseudomonas, Serratia, and Stenotrophomonas, which may engage in beneficial interactions with plants $[4,5]$.

Stenotrophomonas maltophilia is a Gram-negative bacillus found in a wide range of natural habitats, including water sources, soils, rhizospheres, animal microbiotas, including humans $[6,7]$. S. maltophilia has also been used as part of bioremediation and biocontrol strategies $[6,8]$. However, S. maltophilia has been reported as a global multidrug resistant opportunistic pathogen associated with significant mortality rates of up to $37.5 \%$, mainly because of bacteremia and respiratory tract infections in severely debilitated, immunosuppressed or chronic lung disease patients $[9,10]$. S. maltophilia is intrinsically resistant to multiple classes of antibiotics, such as aminoglycosides, carbapenems, and macrolides, posing a therapeutic challenge and delay the administration of proper antibiotics [7].

S. maltophilia present high intraspecific variability [11] and, along with the closely related species S. pavanii, comprise the S. maltophilia complex (Smc) [12-14]. Phylogenic studies based on multilocus sequencing typing (MLST) and whole-genome sequencing revealed the organization of Smc members in several genogroups [15-17]. A study using whole-genome multilocus sequence typing (wgMLST) and average nucleotide identity (ANI) analyses showed the genetic organization of Smc in 23 monophyletic genogroups with different virulence and resistance characteristics [18]. Among those, genogroup 3 ( $\mathrm{Sm} 3$ ) exhibits a myriad of phenotypically distinct strains from different sources. These strains remain poorly explored, hampering investigations on the genetic determinants underlying the physiology and niche occupation of $S$. maltophilia isolates.

Here we present the genome sequencing of $S$. maltophilia UENF-4GII, the first $S$. maltophilia isolated from vermicompost. S. maltophilia UENF-4GII belongs to Sm3 and harbors a set of interesting horizontally-transferred regions, including genomic islands (GIs) involved in phage resistance via DNA modification, and pili glycosylation. ANI analysis and SNP-based phylogenetic reconstructions allowed us to reclassify six publicly available Stenotrophomonas spp. genomes as S. maltophilia from Sm3. We also used the S. maltophilia UENF-4GII genome with those of other 66 S. maltophilia isolates to thoroughly characterize of the population structure, virulence, and resistance profiles of Sm3. Finally, a pangenome-wide association study (pan-GWAS) of Sm3 allowed us to identify genes associated with niche occupation and phylogenetic clusters. 


\section{METHODS}

\section{Bacterial isolation and identification}

The bacterium was isolated from mature manure vermicompost produced from cattle manure at Universidade Estadual do Norte Fluminense Darcy Ribeiro, Brazil. In summary, serial dilutions $\left(10^{-1}\right.$ to $\left.10^{-7}\right)$ were performed on a solution prepared by adding $10 \mathrm{~g}$ of vermicompost in $90 \mathrm{~mL}$ of saline $\left(8.5 \mathrm{~g} \cdot \mathrm{L}^{-1} \mathrm{NaCl}\right)$, followed by shaking for $60 \mathrm{~min}$. Then, $100 \mu \mathrm{L}$ of the final dilution $\left(10^{-7}\right)$ were taken and spread on plates containing solid Nutrient Broth (NB) with $8 \mathrm{~g} \cdot \mathrm{L}^{-1}$ of NB and $15 \mathrm{~g} \cdot \mathrm{L}^{-1}$ of agar in $1 \mathrm{~L}$ of distilled water. After incubation at $30^{\circ} \mathrm{C}$ for 7 days, different colony types could be identified and, for purification, individual colonies were transferred to Petri plates with Dygs solid media acquired from Vetec (São Paulo, Brazil). After isolation and purification on Dygs solid medium, a yellowish, circular, convex elevation, punctiform and smooth surface bacterial colony was selected. Light microscopy revealed a Gram-negative strain and the presence of rod-shaped motile was confirmed under phase contrast microscopy. This isolate, named UENF-4GII, was stored in a $16 \mathrm{~mL}$ glass flask containing $5 \mathrm{~mL}$ of Nutrient Broth solid medium covered with mineral oil and later grown in liquid Dygs medium under rotatory shaker at $150 \mathrm{rpm}$ and $30{ }^{\circ} \mathrm{C}$ for $36 \mathrm{~h}$.

\section{Genome sequencing and annotation}

Genomic DNA was extracted using QIAamp ${ }^{\circledR}$ DNA Mini Kit (Qiagen) and quantified with an Agilent Bioanalyzer 2100 instrument (Agilent, California, USA). Paired-end libraries were previously prepared with the TruSeq Nano DNA LT Library Prep (Illumina) and sequenced on an Illumina HiSeq 2500 sequencing system at the Life Sciences Core Facility (LaCTAD; UNICAMP, Campinas, Brazil). Sequencing reads ( $2 \times 100 \mathrm{bp}$ ) were assembled de novo with SPAdes v.10.3.1 [19] and scaffolded with Gfinisher v.1.4 [20], using as references the complete genome of S. maltophilia JV3 (GCF_000223885.1) and an alternative assembly generated with Velvet 1.2.10 [21]. The assembly statistics were assessed with QUAST v.3.0 [22]. Genome completeness was assessed with BUSCO v.4.0 [23], using the Gammaproteobacteria dataset as reference. PlasmidSpades [24] was used to predict plasmids. The assembled genome was annotated with the NCBI Prokaryotic Genome Annotation Pipeline (PGAP) [25]. The UENF-4GII genome was deposited into DDBJ/EMBL/GenBank under the accession number JABUNQ000000000. Genes involved in antimicrobial metabolite biosynthesis were predicted using antiSMASH [26]. Insertion sequences (ISs) and Gls were predicted with ISEscan v.1.5.4 [27] and Islandviewer4 [28], respectively. Bacteriophage signatures were analyzed with PHASTER [29]. 


\section{Genome similarity assessment}

We downloaded 641 genomes of Stenotrophomonas available in the RefSeq database in January, 2021 [30, 31]. Genome completeness was assessed with BUSCO 4.0 [23], using quality score $\geq 90 \%$ and the Gammaproteobacteria dataset as reference. We excluded assemblies with more than 500 contigs. A preliminary genome distance estimation analysis of isolate UENF-4GII and RefSeq genomes was performed using Mash [32] and a distance tree was generated using MASHtree v.0.50 [33]. All-against-all ANI based on MUMmer alignment (ANIm) was performed with pyani v.0.27 [34]. To assess the concordance between $\mathrm{ANI}$ and Mash estimates, we performed a linear correlation analysis using Pearson's correlation coefficient. For this analysis, the Mash distances were converted into Mash scores (1-Mash distance) to allow a direct comparison with ANI values.

\section{Pangenome analysis}

The Sm3 genogroup pangenome was performed with Roary v.3.6, using 95\% identity threshold to determine orthologous [35]. Core genes were aligned with MAFFT v.7.394 [36]. SNPs were extracted from the core-genome alignment using SNP-sites v.2.3.3 [37] and maximum likelihood phylogenetic reconstructions were performed with IQ-tree [38], with ascertainment bias correction under the model GTR+ASC. The bootstrap support was evaluated using the ultrafast bootstrap method with 1000 replicates [39]. The resulting phylogenetic tree was visualized with iTOL v4 [40].

As a complementary approach, we performed a pangenome-wide association study (pan-GWAS) ) on the on the Sm3 dataset using Scoary [92]. The pan-GWAS was computed using the Roary output to find genes associated with isolation source (clinical or non-clinical) and to establish which genes were typical of each phylogenetic cluster $(\mathrm{PC})$, while correcting for population structure using the core-genome phylogenetic tree (command $-n$ tree). False-discovery rate was estimated by Benjamini-Hochberg adjusted p-value provided in Scoary. We only reported the results with specificity $>70 \%$ and Benjamini-Hochberg corrected $p$-value $<0.05$. The binary heatmaps of traitassociated genes were rendered using $R$ package tidyverse [41].

\section{Virulome and resistome analysis}

Virulence and antimicrobial resistance genes were predicted using Usearch v.11.0.667 screened against the Virulence Factors of Pathogenic Bacteria Database (VFDB) and the Comprehensive Antibiotic Resistance Database (CARD). Minimum identity and coverage thresholds of $60 \%$ and $50 \%$ were used in these searches, respectively. The 
presence/absence profiles of virulence and resistance-associated genes were rendered using R package tidyverse [41].

\section{RESULTS}

\section{Genome analysis of S. maltophilia UENF-4GII}

During the characterization of culturable bacteria from mature cattle vermicompost, we identified a bacterium that was preliminarily characterized as Stenotrophomonas sp. using $16 \mathrm{~S}$ rRNA sequencing. We submitted the genome of this isolate to whole-genome sequencing (see methods for details). The 30,093,445 paired-end reads and were assembled into 3 scaffolds, with an $\mathrm{N}_{50}$ of $1.3 \mathrm{Mbp}$, encompassing a total of $4.4 \mathrm{Mbp}$ with $66.55 \%$ GC content. No plasmids were detected. BUSCO assessment recovered 437 (96.68\%) single copy genes of Gammaprotebacteria dataset, supporting the completeness and high quality of the assembled genome. The genome harbors 3,932 protein-coding genes, 70 tRNA genes, and 2 rRNA operons.

The S. maltophilia UENF-4GII genome carries 18 complete IS elements: nine of the IS481 family, seven of the IS3 family and a single copy of the IS5 and IS21 families (Supplementary figure S1). In addition, we also found an incomplete prophage region that is closely related with a temperate bacteriophage from Burkholderia pseudomallei (Burkho_phi1026b) [42]. AntiSMASH prediction revealed that UENF-4GII possesses four gene clusters involved in the production of two bacteriocin-like compounds, an aryl polyene related to Xanthomonadin [43], and a non-ribosomal peptide synthetase (NRPS) gene cluster involved in the biosynthesis of siderophore (Supplementary figure S1). Siderophores are the most important iron uptake systems in S. maltophilia [44]. In NRPS gene cluster, we identified six genes (HRE58_11480 to 11500, and HRE58_11505) encoding an specific siderophore of S. maltophilia [45]. These enzymes are similar to those involved in enterobactin biosynthesis in enteric bacteria (e.g. E. coli) [44].

\section{Genome similarity of UENF-4GII}

In order to investigate the genomic relatedness of UENF-4GII strain, we downloaded all available Stenotrophomonas genomes in Refseq database ( $n=641$, January 2021), out of which 627 were retained after BUSCO quality filtering (see method for details). Genome identity was computed using Mash and ANI. The mash-distance phylogeny highlights the diversity of the Stenotrophomonas genus, corroborating the S. maltophilia complex organization in different genogroups (Figure 1A), as previously described by Gröschel et al. [18]. We identified 17 genogroups according to the distance estimation of wellcharacterized representative genomes, including the affiliation of UENF-4GII to Sm3, along with other 67 clinical and non-clinical strains (Supplementary table S1). In 
addition, this analysis allowed us to reclassify six Stenotrophomonas spp. as $S$. maltophilia Sm3 isolates, including the environmental strains Stenotrophomonas sp. DDT-1 (GCF_001580555.1) and Stenotrophomonas sp. Pemsol (GCF_003586545.1), reported as specific biodegraders of organochlorine pesticide (DDT) and polycyclic aromatic hydrocarbons (PAH), respectively. Mash results are supported by ANI analysis, which showed genomic identity above 95\% within the same genogroup (Figure 1B). Moreover, the strong positive linear correlation ( $r=0.98$, $p$-value $<2.2 \mathrm{e}-16)$ between $\mathrm{ANI}$ and Mash results (Figure 1C) confirm a similar level of resolution for species delineation within the Stenotrophomonas genus.

\section{Pangenome analysis}

The pangenome analysis of the $67 \mathrm{Sm} 3$ isolates comprises 13,380 genes, with 2,754 core genes (i.e. present in at least $95 \%$ of the strains), 1,958 shell genes (present in $15 \%$ to $95 \%$ of the strains), and 8,668 cloud genes (present in up to $15 \%$ of the strains). The heap law estimate supports an open pangenome (alpha=0.65) (Figure $2 \mathrm{~A}$ ), which typically reflects a high genetic diversity through the acquisition of exogenous DNA [46]. The genomic fluidity $(\phi) \mathrm{Sm} 3$ was estimated in 0.18 ( \pm 0.05 ), indicating that an average of $18 \%$ of unique gene families between in a given pair of genomes.

We also performed a maximum-likelihood phylogenetic reconstruction using SNPs extracted from core genes (i.e. core-genome multilocus sequencing typing, cgMLST). This analysis revealed that Sm3 comprises three distinct and highly supported phylogenetic clusters (PC) (Figure 2B). PC1 $(n=21)$ and PC2 $(n=15)$ encompass clinical and non-clinical strains, while PC3 $(n=31)$ has only clinical strains. Interestingly, 10 of the 21 PC1 strains are non-clinical, including S. maltophilia UENF-4GII.

We identified 75 unique genes in S. maltophilia UENF-4GII (Supplementary table S2), including pbpX (HRE58_12720), which encodes a low molecular weight penicillinbinding protein (LMW-PBP). This gene is closely located to other unique genes: a gene with unknown function (HRE58_12725) and two transposase genes (HRE58_12730, HRE58_12735), flanked by a perfect 26-bp inverted repeat (IR) sequence, supporting their acquisition by horizontal gene transfer (Supplementary figure 2). LMW-PBPs are important enzymes involved in cell-wall recycling and considered the major molecular targets for $\beta$-lactam antibiotics [47]. Alterations in the structure of LMW-PBPs are associated with reduced susceptibility to penicillin and other $\beta$-lactams and can induce the expression of $\beta$-lactamases [47-49]. The presence of an LMW-PBP composite transposon likely confers a competitive advantage for S. maltophilia UENF-4GII to thrive in microbial communities containing penicillin.

\section{UENF-4GII genomic islands}


To further understand genome plasticity, we identified and analyzed 17 putative Gls (GI1-17) in S. maltophilia UENF-4GII, which probably represent recent horizontal gene transfers (Supplementary table S3). In addition, we compared these Gls with other Sm3 genomes (Figure 3). Among these regions, GI3 harbors an operon (HRE58_02625-02660) containing genes encoding GalE, HIdD, and glycosyltransferases involved in lipopolysaccharide biosynthesis. This operon is present in 68\% of the PC3 genomes. GI16 contains a class $\mathrm{C}$ beta-lactamase and $\mathrm{GI} 10$ have blue-light sensor genes that are absent in PC3 genomes.

Interestingly, $60 \%$ of the $S$. maltophilia UENF-4GII unique genes are located within 7 Gls related with DNA metabolism (GI6 and GI17), virulence (GI7 and GI15) and unknown functions (GI2, GI9 and GI14). GI6 has 9 unique genes, including a restrictionmodification (R-M) system with two type I restriction endonucleases (HRE58_05925 and HRE58_05935) and a DNA methyltransferase (HRE58_05920). R-M systems defend the bacterial genomes against bacteriophages and other types of exogenous genetic elements [50] and, hence, their acquisition might be adaptive by boosting resistance against phages [51, 52]. The GI17 has 41,433 bp and comprises 14 unique genes, including a DNA polymerase-like protein (HRE58_18840) and an operon containing an 8-oxoguanine DNA glycosylase (HRE58_18870), a nitroreductase (HRE58_18875), a 7cyano-7-deazaguanine synthase (HRE58_18880; PreQ(0)), and a nucleoside 2deoxyribosyltransferase (HRE58_18885). These genes are likely part of a repair system for oxidative stress-mediated DNA damage [53]. PreQ(0) might be involved in the insertion of 7-deazaguanine derivatives in the DNA [54], as part of a defense system against foreign DNA and phages [55].

Finally, 9 of 10 genes from GI15 are unique (Supplementary table S3). This island is involved in the biosynthesis and glycosylation of type IV pilin-like proteins. Hence, this island is likely an important virulence factor that mediate bacterial adherence to biotic and abiotic surfaces. Although pilin glycosylation has been associated with immune evasion of pathogenic bacteria [56], a recent study showed its role in the defense against phage infection [57]. Collectively, these GIs indicate that S. maltophilia UENF-4GII is equipped with different horizontally-acquired genes that increased its tolerance to foreign DNA.

\section{Pan-GWAS of Sm3 genogroup}

We performed a pan-GWAS to identify genes associated with environment type (clinical and non-clinical; Supplementary table S1) and PCs in Sm3 using Scoary. We found 299 and 172 gene clusters associated with clinical and non-clinical environments, respectively (Supplementary figure S3, Supplementary table S4). Interestingly, 58\% of the 471 environment-associated genes encode hypothetical proteins with unknown function. 
Among the genes associated with clinical environments, we identified genes encoding a chemotaxis response regulator protein (cheB), blue-light sensing protein (b/uF), and trehalose sintase tres, a crucial enzyme involved in trehalose biosynthesis. Several bacteria use trehalose as carbon source and stress protectant [58]. Trehalose metabolism has also been associated with the emergence of virulent human pathogens $[59,60]$. Interestingly, tres was also reported as a significant gene associated with clinical strains of the S. maltophilia complex [61]. Moreover, we identified significant associations with genes involved in bacterial resistance, including multidrug efflux pumps ( $m d t \mathrm{~A}$ and $m d t C$ ), macrolide efflux (macA), aminoglycoside-modifying enzyme (aac), $\beta$-lactamases (blaL1 and blaL2), and a $\beta$-lactam sensor gene (b/aR1). BlaL1 and BlaL2 are well known metallo- $\beta$-lactamases in Stenotrophomonas. They hydrolyze almost all $\beta$-lactams, are resistant to all clinically available $\beta$-lactamase inhibitors [62, 63 ] and have been show to increase pathogenicity in clinical settings [63, 64]. Among the genes associated with non-clinical environments, we also found genes involved in bacterial resistance, particularly in macrolide efflux (macA and macB), as well as three gene clusters involved in heavy metal resistance including copper (copB), cobalt/zinc/cadmium (czcB), and mercury (merR1).

As most non-clinical strains belong to PC1 and PC3 comprised only clinical strains, most of the environment-related genes are also linked with their respective phylogenetic groups. A total of 252, 97 and 442 gene clusters were associated, respectively, with PC1, PC2 and PC3 (Figure 4, Supplementary table S5). The majority (60\%) of the PC-associated genes encoded proteins with unknown functions. PC1 was strongly associated ( $100 \%$ of sensitivity and specificity) with 13 gene clusters, including a type II toxin-antitoxin gene (pasl) and c-AMP phosphodiesterase encoding gene (cpdA) involved in stress resistance $[65,66]$. Further, 11 gene clusters were strongly associated to $\mathrm{PC2}$, including besA, involved in iron metabolism [67], and katG, related with oxidative stress resistance [68]. The PC3 presented 107 associated gene clusters, with $100 \%$ sensitivity and specificity. Among these, we identified genes involved in transcriptional regulation (cueR, com R, rstA, and $c y s \mathrm{~L}$ ), iron metabolism (besA), antibiotic efflux (mdtA and $m d t C$ ), and $\beta$-lactam resistance (bla $\mathrm{L} 1$ ).

The variants found in different traits (e.g. besA, macA and blaL1) are directly associated with genomic diversity of the PCs. A closer look into the blaL1 variant of PC3 reveals a molecular heterogeneity with $86 \%$ mean similarity with blaL1 from PC1 and PC2 genomes. Although the functional implications of such diversity is not fully understood, variation in blaL1 have been associated with $\beta$-lactam resistance in $S$. maltophilia, which may contribute to its increased prevalence as a nosocomial pathogen $[63,64,69]$.

\section{Virulome and resistome analysis of the $\mathrm{Sm} 3$ genogroup}


In order to understand the pathogenic potential within the Sm3 genogroup, we systematically investigated the distribution of virulence and antimicrobial resistance genes. The Sm3 virulome contains 54 genes (Supplementary table S6), out of which 35 constitute the core virulome. Most (21 out of 35) of the core virulence genes are involved in motility and adherence, including pilMNOPQ and the pi/TU operon, which encode type IV pili subunits [70], and tuf (elongation factor Tu), involved in adhesion to host cells and extracellular matrix components [71]. In addition, we also identified seven type II secretion system (T2SS) genes (e.g. xpsEFG and $h x c R S$ ), which promote the export of enzymes during bacterial colonization. Further, the presence of T2SS genes along with other core virulence genes (e.g. $\operatorname{acpXI}$, hemB, hemL, csrA, and $i c l)$, can be involved in infection and immune evasion capacity of Sm3 strains.

The Sm3 accessory virulome comprises 12 low and 7 high frequency genes, respectively (Figure 5). The high frequency genes are associated with adherence (pin $\mathrm{N}$ ), motility $(\mathrm{f} / \mathrm{mH})$, iron uptake (bauA), immune evasion $(\mathrm{rm} / \mathrm{B})$, and stress tolerance $(\mathrm{c} / \mathrm{pB}$, $k a t \mathrm{~A}$, and $k a t \mathrm{G})$. The $c / p \mathrm{~B}$ gene encodes a heat shock-inducible chaperone required for bacterial tolerance to a variety of stresses, including heat, osmotic and acidic stress [72]. The katA gene encodes a catalase that might confer tolerance against hydrogen peroxide-based disinfectants $[18,73]$ and is absent in all PC2 strains. Nevertheless, PC2 has an alternative catalase-peroxidase gene (katG) that is absent in PC1 and PC3. The katG gene is associated with oxidative stress control and has different roles in pathogenic bacteria $[68,74]$. Four catalases (KatA1, KatA2, KatMn, and KatE) have been described in S. maltophilia, out of which KatA has been considered the main factor in conferring tolerance against hydrogen peroxide [75]. The apparent displacement of katA by katG in PC2 warrants further investigation and might provide insights the response of S. maltophilia to oxidative stress.

The resistome analysis revealed 23 antimicrobial resistance genes (Supplementary table S7), out of which 17 comprise the core resistome, including the metallo- $\beta$-lactamase BlaL1 and the inducible Ambler class A $\beta$-lactamase BlaL2, as described above [76]. Further, antibiotic efflux pumps (e.g. SmeABC, SmeDEF, SmeRS, OqxAB, GolS, EmrE, and MexK) represent the main resistance mechanism encoded by the $\mathrm{Sm} 3$ core resistome.

The accessory resistome encompasses six genes (Figure 5). No accessory genes were found in PC2, as well as in most PC1 strains, including S. maltophilia UENF-4GII. The accessory resistome contains two classes of aminoglycoside-modifying enzymes: aminoglycoside phosphotransferase (encoded by $a p h(6)-\mathrm{Id}$ and $\left.a p h\left(3^{\prime \prime}\right) \mid \mathrm{b}\right)$; and $\mathrm{N}$ aminoglycoside acetyltransferase (encoded by $\operatorname{aac}\left(6^{\prime}\right)$-lz and $\left.a a c\left(6^{\prime}\right)-\mathrm{lak}\right)$. The $\operatorname{aac}\left(6^{\prime}\right)-\mathrm{Iz}$ gene was exclusively found in 25 (81\%) PC3 genomes, whereas aac ( $\left.6^{\prime}\right)$-lak and aph genes were found in four PC1 genomes. Only two strains (G4S2 and G4S2-1) have the sulfonamide resistance gene sul 2 and the chloramphenicol resistance gene floR. In summary, the Sm3 genomes do not present a large repertoire of acquired resistance 


\section{6}

genes. The low frequency of sulfonamide resistance genes suggests that this antibiotic class might be a good therapeutic strategy against $S$. maltophilia infections, as previously proposed [18].

\section{CONCLUSION}

In this study, we report the sequencing and in-depth analysis of the S. maltophilia UENF4GII genome. This genome harbors a range of exclusive Gls associated with DNAmodification and anti-phage defense systems. Comparative analysis using pairwise genome identity metrics and cgMLST phylogeny show that S. maltophilia UENF-4GII belong to the Sm3 genogroup, which comprises three PCs. Using a pan-GWAS approach, we identified 131 genes as significantly associated with specific Sm3 PCs. Further, 471 genes were specifically associated with clinical and non-clinical environments. These genes could be used as biomarkers in future studies. Sm3 genomes comprise a low number of acquired virulence and resistance genes, although the presence of kat $\mathrm{G}$ and aminoglycoside resistance genes were associated with specific PCs. Collectively, our results provide important information regarding $S$. maltophilia genomic diversity that could provide the grounds for more detailed clinical and ecological investigations.

\section{ACKNOWLEDGEMENTS}

This work was supported by Fundação Carlos Chagas Filho de Amparo à Pesquisa do Estado do Rio de Janeiro (FAPERJ; grants E-26/203.309/2016 and E-26/203.014/2018), Coordenação de Aperfeiçoamento de Pessoal de Nível Superior - Brasil (CAPES; Finance Code 001), and Conselho Nacional de Desenvolvimento Científico e Tecnológico. The funding agencies had no role in the design of the study and collection, analysis, and interpretation of data and in writing.

\section{AUTHOR CONTRIBUTIONS}

Conceived the study: FP-S, TMV, FLO; Funding and resources: TMV, FLO; Data analysis: FP-S, HP-A; Interpretation of the results: FP-S, FPM, HP-A, TMV; Wrote the manuscript: FP-S, FPM, HP-A, TMV. 
A
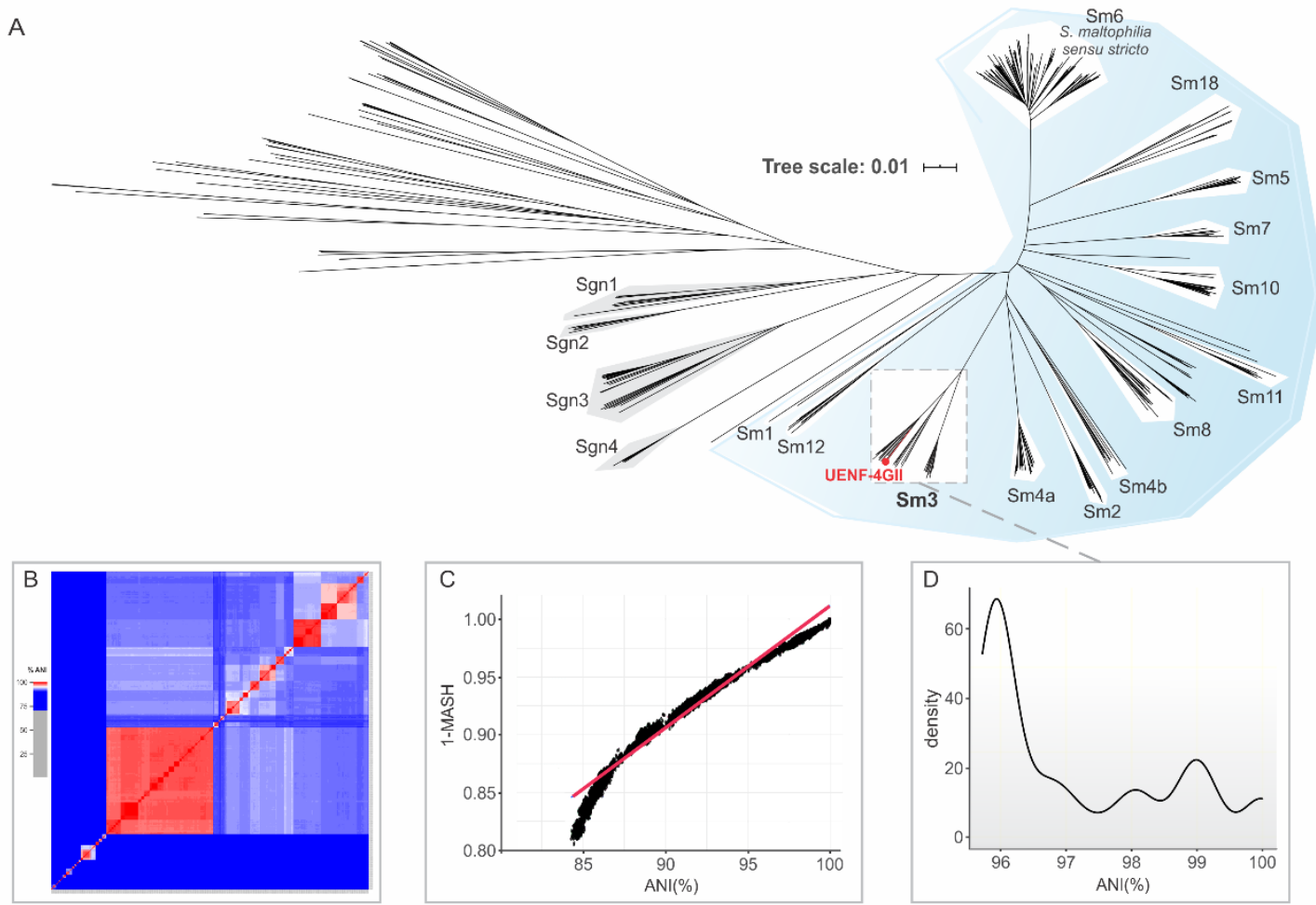

385

Figure 1. Genomic diversity of Stenotrophomonas genus. (A) Mash-distance-based phylogeny of Stenotrophomonas, built using 627 publicly genomes and that of UENF4GII (red color). The S. maltophilia sensu lato clade is shaded in light blue. (B) Pairwise average nucleotide identity (ANI) calculated with 627 Stenotrophomonas genomes. Colors depict the degree of genome identity. (C) Correlation between ANI and Mash methods. (D) Density plot of pairwise ANI within the Sm3 genogroup. 
A

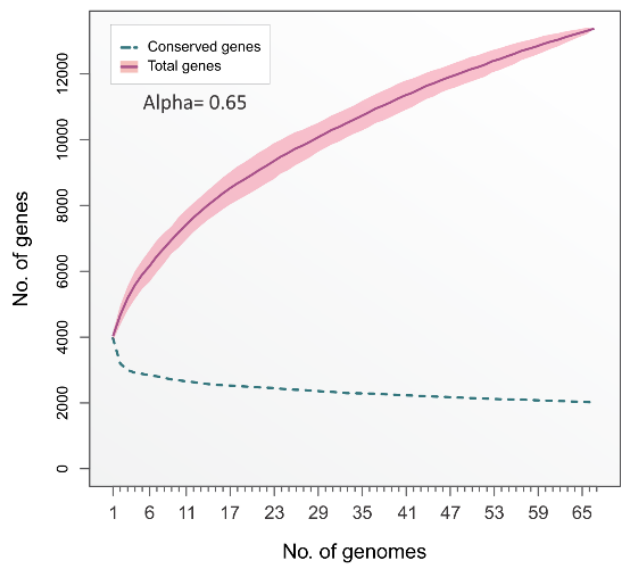

B

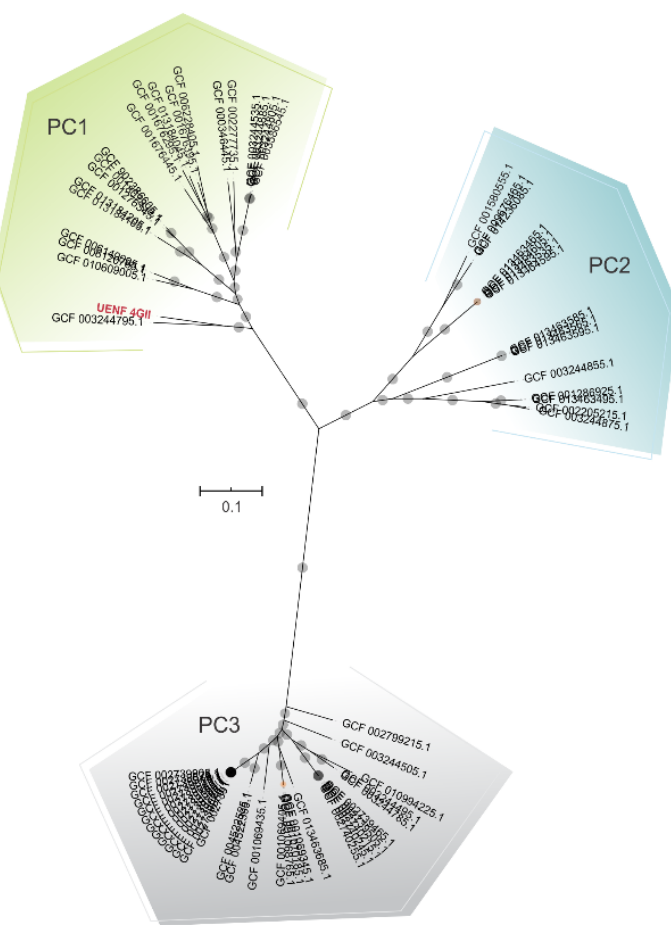

394 Figure 2. Pangenome and phylogeny of Sm3 genogroup. (A) Number of gene families in the Sm3 pangenome. The cumulative curve (in dark-red) and alpha value of the Heap law less than one (0.65) supports an open pangenome. (B) cgMLST of Sm3 genogroup showing three phylogenetic groups. SNPs extracted from the core genome were used to build a maximum likelihood phylogenetic tree using IQ-tree (see methods for details). Bootstrap values below and above $70 \%$ are represented by orange and gray points, respectively. 


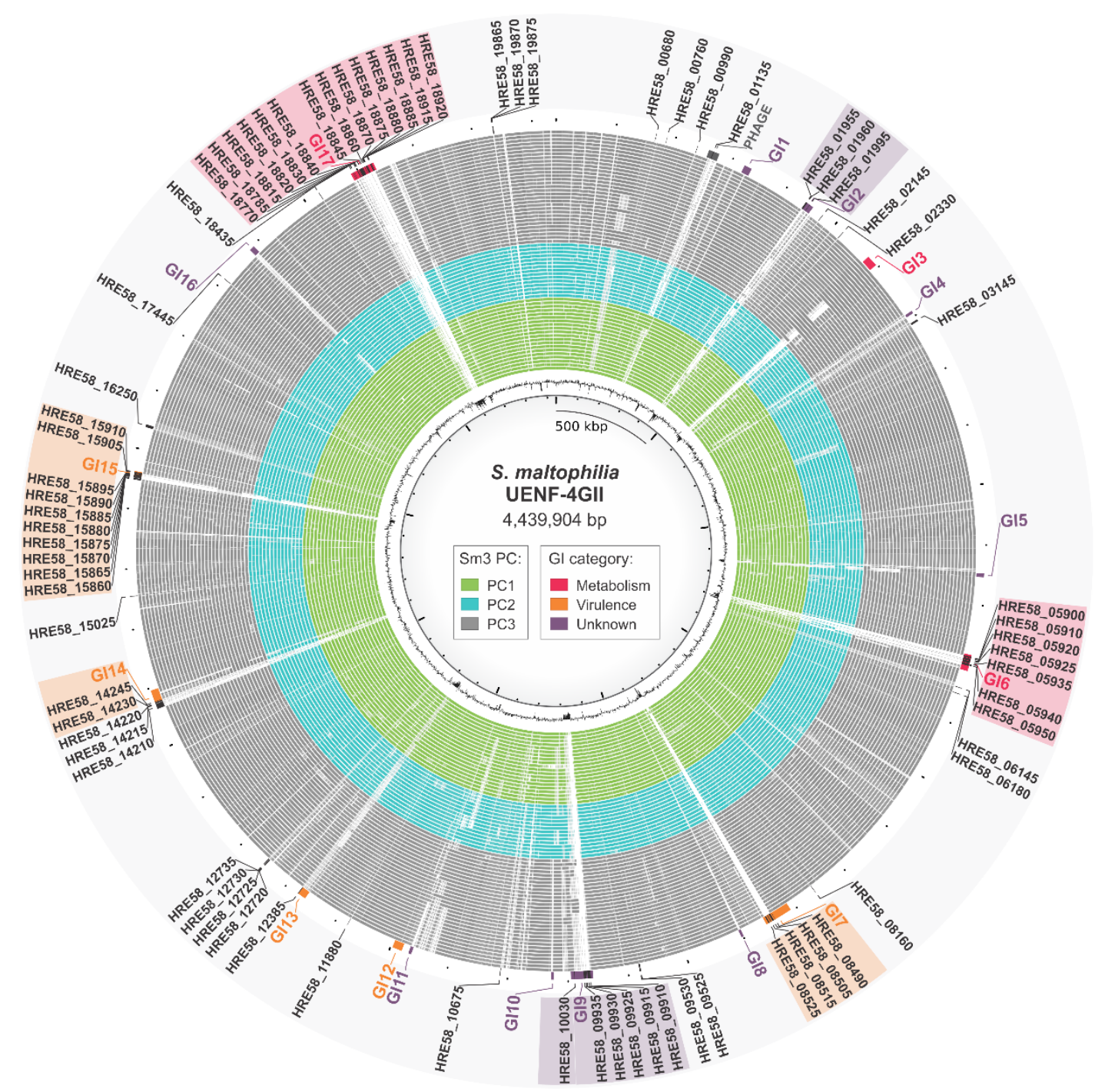

401

402 Figure 3. Circular genome representation of 75 unique genes and $\mathbf{1 7}$ genomic islands 403 (GIs) identified in S. maltophilia UENF-4GII in comparison to other $66 \mathrm{Sm} 3$ strains. The 404 inner ring represents the UENF-4GII genome GC content. Green, blue and gray rings represent genomes from PC1, PC2 and PC3, respectively. The Gls were predicted and classified as metabolic (red), virulence (orange) or unknown (purple). Unique genes predicted within Gls were highlighted according to the GI category. 

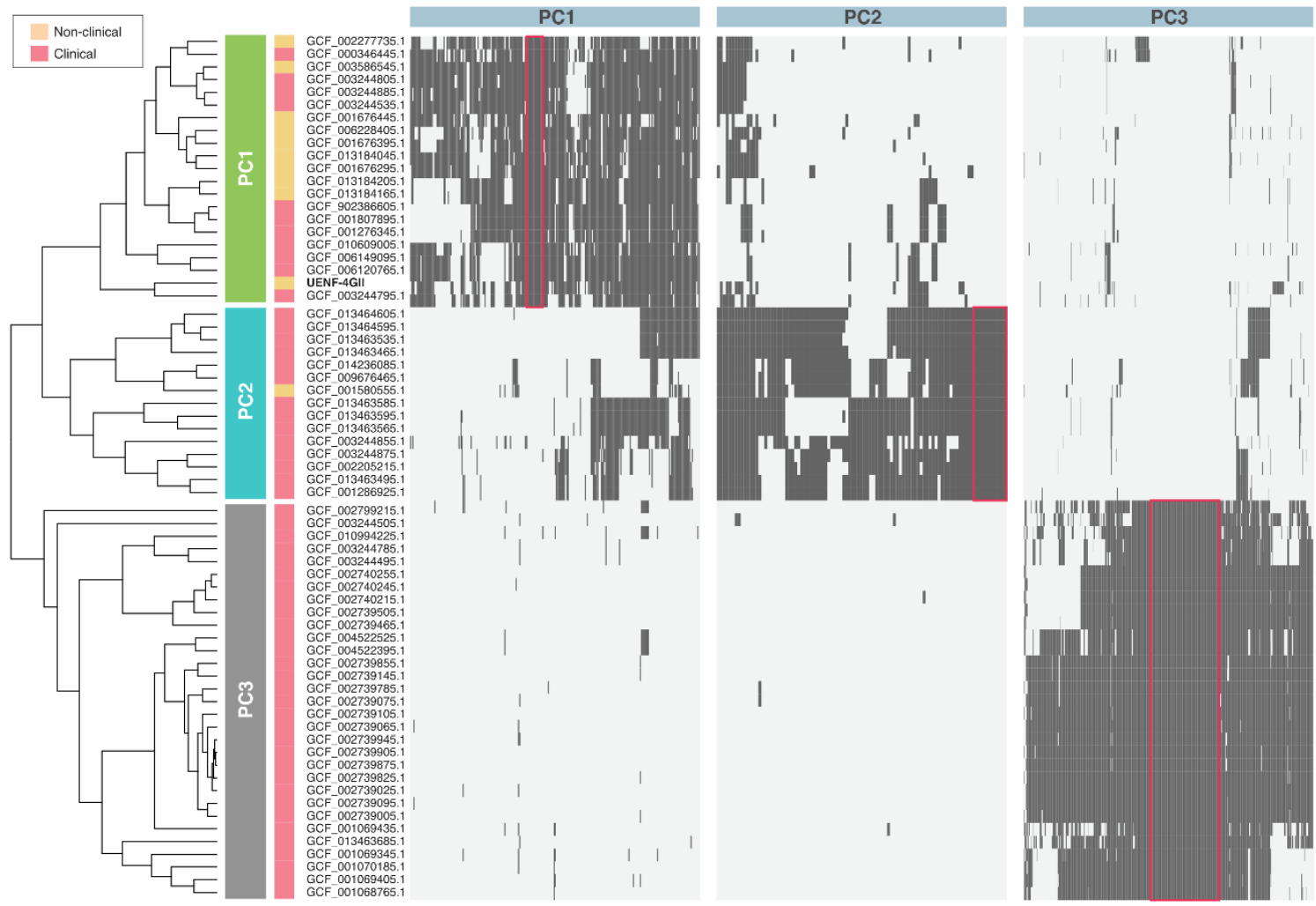

409 Figure 4. Distribution of PC-associated genes in S. maltophilia Sm3 genogroup. The

410 cgMLST tree is annotated with two strips representing the phylogenetic cluster (PC) and 411 the environment source. The heatmaps represent the presence (dark-gray) or absence 412 (light-gray) of the genes identified by the pan-GWAS pipeline using phylogenetic cluster $413(\mathrm{PC})$ as trait. The neon red square highlights the most strongly associated genes found 414 for each trait (i.e. 100\% specificity and sensitivity). 


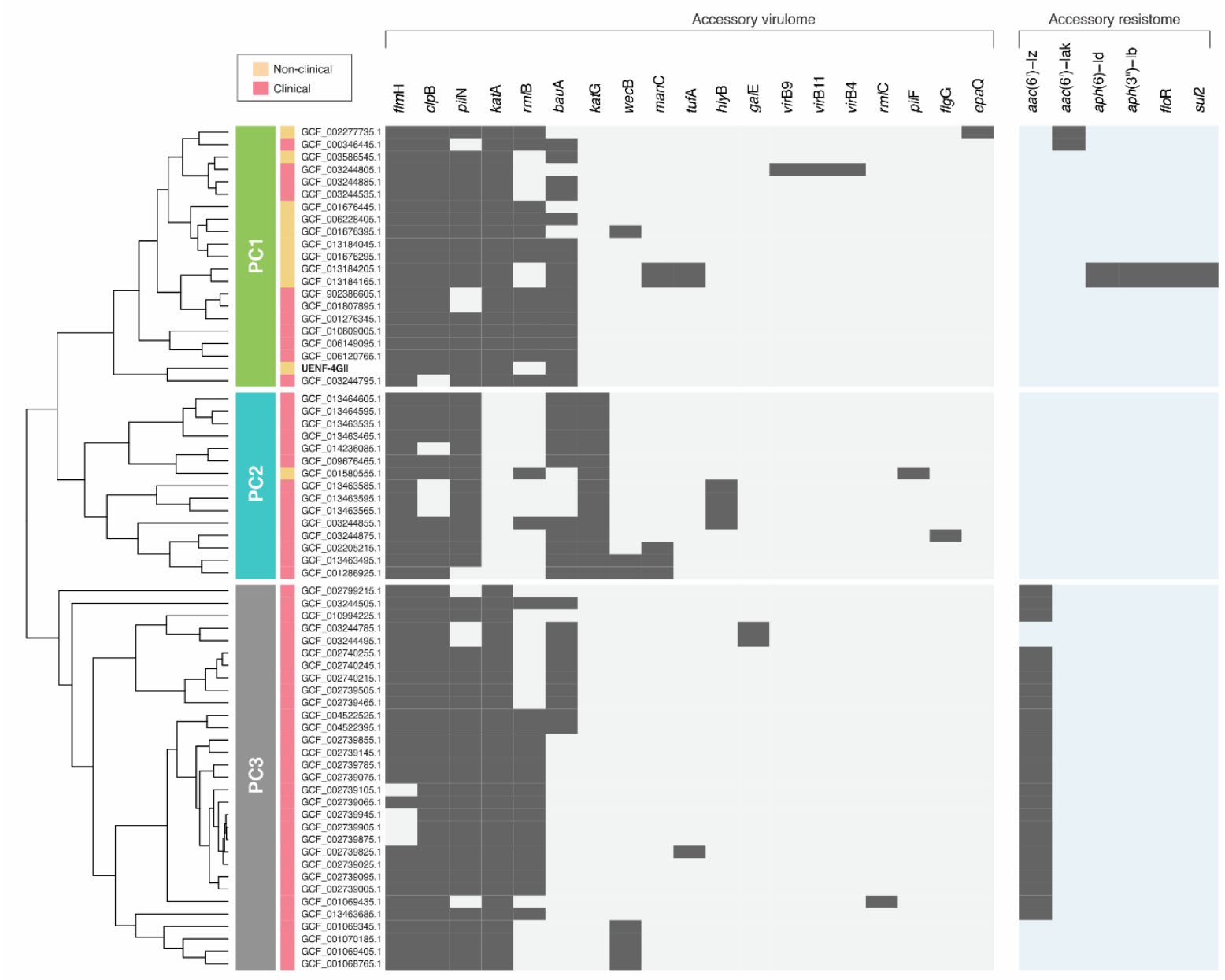

Figure 5. Acquired virulome and resistome of S. maltophilia Sm3 genogroup. The cgMLST tree is annotated with two strips representing the phylogenetic cluster (PC) identified and the environment source. The binary heatmaps represent the presence (dark-gray) or absence (light-gray/light-blue) of the genes identified.

\section{REFERENCES}

1. Lim SL, Lee LH, Wu TY: Sustainability of using composting and vermicomposting technologies for organic solid waste biotransformation: recent overview, greenhouse gases emissions and economic analysis. Journal of Cleaner Production 2016, 111:262278.

2. Domínguez J, Aira M, Kolbe AR, Gómez-Brandón M, Pérez-Losada M: Changes in the composition and function of bacterial communities during vermicomposting may explain beneficial properties of vermicompost. Scientific reports 2019, 9(1):1-11.

3. Pathma J, Sakthivel N: Microbial diversity of vermicompost bacteria that exhibit useful agricultural traits and waste management potential. SpringerPlus 2012, 1(1):26.

4. Pathma J, Sakthivel N: Molecular and functional characterization of bacteria isolated from straw and goat manure based vermicompost. Applied soil ecology 2013, 70:3347. 
5. Matteoli FP, Passarelli-Araujo H, Reis RJA, da Rocha LO, de Souza EM, Aravind L, Olivares FL, Venancio TM: Genome sequencing and assessment of plant growthpromoting properties of a Serratia marcescens strain isolated from vermicompost. BMC Genomics 2018, 19(n. 1):750.

6. Ryan RP, Monchy S, Cardinale M, Taghavi S, Crossman L, Avison MB, Berg G, Van Der Lelie $D$, Dow JM: The versatility and adaptation of bacteria from the genus Stenotrophomonas. Nature Reviews Microbiology 2009, 7(7):514-525.

7. Brooke JS: Stenotrophomonas maltophilia: an emerging global opportunistic pathogen. Clinical microbiology reviews 2012, 25(1):2-41.

8. Mukherjee P, Roy P: Genomic potential of Stenotrophomonas maltophilia in bioremediation with an assessment of its multifaceted role in our environment. Frontiers in Microbiology 2016, 7:967.

9. Falagas ME, Kastoris AC, Vouloumanou EK, Rafailidis PI, Kapaskelis AM, Dimopoulos G: Attributable mortality of Stenotrophomonas maltophilia infections: a systematic review of the literature. Future microbiology 2009, 4(9):1103-1109.

10. Zemanick ET, Wagner BD, Robertson CE, Ahrens RC, Chmiel JF, Clancy JP, Gibson RL, Harris WT, Kurland G, Laguna TA: Airway microbiota across age and disease spectrum in cystic fibrosis. European Respiratory Journal 2017, 50(5).

11. Gherardi G, Creti R, Pompilio A, Di Bonaventura G: An overview of various typing methods for clinical epidemiology of the emerging pathogen Stenotrophomonas maltophilia. Diagnostic microbiology and infectious disease 2015, 81(3):219-226.

12. Rhee J-Y, Choi JY, Choi M-J, Song J-H, Peck KR, Ko KS: Distinct groups and antimicrobial resistance of clinical Stenotrophomonas maltophilia complex isolates from Korea. Journal of medical microbiology 2013, 62(5):748-753.

13. Hauben L, Vauterin L, Moore E, Hoste B, Swings J: Genomic diversity of the genus Stenotrophomonas. International Journal of Systematic and Evolutionary Microbiology 1999, 49(4):1749-1760.

14. Svensson-Stadler LA, Mihaylova SA, Moore ER: Stenotrophomonas interspecies differentiation and identification by gyrB sequence analysis. FEMS microbiology letters 2012, 327(1):15-24.

15. Ochoa-Sánchez LE, Vinuesa P: Evolutionary genetic analysis uncovers multiple species with distinct habitat preferences and antibiotic resistance phenotypes in the Stenotrophomonas maltophilia complex. Frontiers in microbiology 2017, 8:1548.

16. Patil PP, Kumar S, Midha S, Gautam V, Patil PB: Taxonogenomics reveal multiple novel genomospecies associated with clinical isolates of Stenotrophomonas maltophilia. Microbial genomics 2018, 4(8).

17. Steinmann J, Mamat U, Abda EM, Kirchhoff L, Streit WR, Schaible UE, Niemann S, Kohl TA: Analysis of phylogenetic variation of Stenotrophomonas maltophilia reveals human-specific branches. Frontiers in microbiology 2018, 9:806.

18. Gröschel MI, Meehan CJ, Barilar I, Diricks M, Gonzaga A, Steglich M, Conchillo-Solé O, Scherer I-C, Mamat U, Luz CF: The phylogenetic landscape and nosocomial spread of the multidrug-resistant opportunist Stenotrophomonas maltophilia. Nature Communications 2020, 11(1):1-12.

19. Bankevich A, Nurk S, Antipov D, Gurevich AA, Dvorkin M, Kulikov AS, Lesin VM, Nikolenko SI, Pham S, Prjibelski AD et al: SPAdes: a new genome assembly algorithm and its applications to single-cell sequencing. Journal of computational biology : a journal of computational molecular cell biology 2012, 19(5):455-477.

20. Guizelini D, Raittz RT, Cruz LM, Souza EM, Steffens MB, Pedrosa FO: GFinisher: a new strategy to refine and finish bacterial genome assemblies. Scientific reports 2016, 6:34963.

21. Zerbino R, Birney E: Velvet: algorithms for de novo short read assembly using de Bruijn graphs, vol. 18; 2008. 
22. Gurevich A, Saveliev V, Vyahhi N, Tesler G: QUAST: quality assessment tool for genome assemblies. Bioinformatics 2013, 29(8):1072-1075.

23. Simão FA, Waterhouse RM, loannidis P, Kriventseva EV, Zdobnov EM: BUSCO: assessing genome assembly and annotation completeness with single-copy orthologs. Bioinformatics 2015, 31(19):3210-3212.

24. Antipov D, Hartwick N, Shen M, Raiko M, Lapidus A, Pevzner PA: plasmidSPAdes: assembling plasmids from whole genome sequencing data. Bioinformatics 2016, 32(22):3380-3387.

25. Tatusova T, DiCuccio M, Badretdin A, Chetvernin V, Nawrocki EP, Zaslavsky L, Lomsadze A, Pruitt KD, Borodovsky M, Ostell J: NCBI prokaryotic genome annotation pipeline. Nucleic acids research 2016, 44(14):6614-6624.

26. Medema MH, Blin K, Cimermancic $P$, de Jager V, Zakrzewski $P$, Fischbach MA, Weber T, Takano $E$, Breitling R: antiSMASH: rapid identification, annotation and analysis of secondary metabolite biosynthesis gene clusters in bacterial and fungal genome sequences. Nucleic acids research 2011, 39(suppl_2):W339-W346.

27. Xie Z, Tang H: ISEScan: automated identification of insertion sequence elements in prokaryotic genomes. Bioinformatics 2017, 33(21):3340-3347.

28. Bertelli C, Laird MR, Williams KP, Group SFURC, Lau BY, Hoad G, Winsor GL, Brinkman FS: IslandViewer 4: expanded prediction of genomic islands for larger-scale datasets. Nucleic acids research 2017, 45(W1):W30-W35.

29. Arndt D, Grant JR, Marcu A, Sajed T, Pon A, Liang Y, Wishart DS: PHASTER: a better, faster version of the PHAST phage search tool. Nucleic acids research 2016, 44(W1):W16-W21.

30. O'Leary NA, Wright MW, Brister JR, Ciufo S, Haddad D, McVeigh R, Rajput B, Robbertse $B$, Smith-White B, Ako-Adjei D et al: Reference sequence (RefSeq) database at NCBI: current status, taxonomic expansion, and functional annotation. Nucleic Acids Res 2016, 44(D1):D733-745.

31. Pruitt KD, Tatusova T, Maglott DR: NCBI reference sequences (RefSeq): a curated nonredundant sequence database of genomes, transcripts and proteins. Nucleic Acids Res 2007, 35(Database issue):D61-65.

32. Ondov BD, Treangen TJ, Melsted P, Mallonee AB, Bergman NH, Koren S, Phillippy AM: Mash: fast genome and metagenome distance estimation using MinHash. Genome Biology 2016, 17(n. 1):132.

33. Katz L, Griswold T, Carleton H: Generating WGS Trees with Mashtree. In: Poster presented at: American Society for Microbiology Conference on Rapid Applied Microbial Next-Generation Sequencing and Bioinformatic Pipelines: 2017; 2017: 8-11.

34. Pritchard L, Glover RH, Humphris S, Elphinstone JG, Toth IK: Genomics and taxonomy in diagnostics for food security: soft-rotting enterobacterial plant pathogens. Analytical Methods 2016, 8(1):12-24.

35. Page AJ, Cummins CA, Hunt M, Wong VK, Reuter S, Holden MT, Fookes M, Falush D, Keane JA, Parkhill J: Roary: rapid large-scale prokaryote pan genome analysis. Bioinformatics 2015, 31(22):3691-3693.

36. Katoh $\mathrm{K}$, Standley DM: MAFFT multiple sequence alignment software version 7: improvements in performance and usability. Molecular biology and evolution 2013, 30(4):772-780.

37. Page AJ, Taylor B, Delaney AJ, Soares J, Seemann T, Keane JA, Harris SR: SNP-sites: rapid efficient extraction of SNPs from multi-FASTA alignments. Microbial Genomics 2016, 2(4).

38. Nguyen L-T, Schmidt HA, von Haeseler A, Minh BQ: IQ-TREE: a fast and effective stochastic algorithm for estimating maximum-likelihood phylogenies. Molecular biology and evolution 2014, 32(1):268-274. 
39. Minh BQ, Nguyen MAT, von Haeseler A: Ultrafast approximation for phylogenetic bootstrap. Molecular biology and evolution 2013, 30(5):1188-1195.

40. Letunic I, Bork P: Interactive Tree Of Life (iTOL) v4: recent updates and new developments. Nucleic acids research 2019.

41. Wickham H, Averick M, Bryan J, Chang W, McGowan LDA, François R, Grolemund G, Hayes A, Henry L, Hester J: Welcome to the Tidyverse. Journal of Open Source Software 2019, 4(43):1686.

42. Yuzenkova J, Nechaev S, Berlin J, Rogulja D, Kuznedelov K, Inman R, Mushegian A, Severinov K: Genome of Xanthomonas oryzae bacteriophage Xp10: an odd T-odd phage. Journal of molecular biology 2003, 330(4):735-748.

43. Goel AK, Rajagopal L, Nagesh N, Sonti RV: Genetic locus encoding functions involved in biosynthesis and outer membrane localization of xanthomonadin in Xanthomonas oryzae pv. oryzae. Journal of bacteriology 2002, 184(13):3539-3548.

44. Kalidasan V, Joseph N, Kumar S, Awang Hamat R, Neela VK: Iron and virulence in Stenotrophomonas maltophilia: all we know so far. Frontiers in cellular and infection microbiology 2018, 8:401.

45. Nas MY, Cianciotto NP: Stenotrophomonas maltophilia produces an EntC-dependent catecholate siderophore that is distinct from enterobactin. Microbiology 2017, 163(11):1590.

46. Tettelin H, Medini D: The pangenome: Diversity, dynamics and evolution of genomes. 2020.

47. Ealand CS, Machowski EE, Kana BD: $\beta$-lactam resistance: The role of low molecular weight penicillin binding proteins, $\beta$-lactamases and Id-transpeptidases in bacteria associated with respiratory tract infections. IUBMB life 2018, 70(9):855-868.

48. Sarkar SK, Chowdhury C, Ghosh AS: Deletion of penicillin-binding protein 5 (PBP5) sensitises Escherichia coli cells to $\beta$-lactam agents. International journal of antimicrobial agents 2010, 35(3):244-249.

49. Huang $Y-W$, Wang $Y$, Lin $Y$, Lin C, Lin $Y-T$, Hsu C-C, Yang T-C: Impacts of penicillin binding protein $\mathbf{2}$ inactivation on $\beta$-lactamase expression and muropeptide profile in Stenotrophomonas maltophilia. Msystems 2017, 2(4).

50. Dimitriu T, Szczelkun MD, Westra ER: Evolutionary Ecology and Interplay of Prokaryotic Innate and Adaptive Immune Systems. Current Biology 2020, 30(19):R1189-R1202.

51. Zheng Y, Cohen-Karni D, Xu D, Chin HG, Wilson G, Pradhan S, Roberts RJ: A unique family of Mrr-like modification-dependent restriction endonucleases. Nucleic acids research 2010, 38(16):5527-5534.

52. Tock MR, Dryden DT: The biology of restriction and anti-restriction. Current opinion in microbiology 2005, 8(4):466-472.

53. David SS, O'Shea VL, Kundu S: Base-excision repair of oxidative DNA damage. Nature 2007, 447(7147):941-950.

54. Hutinet G, Swarjo MA, de Crécy-Lagard V: Deazaguanine derivatives, examples of crosstalk between RNA and DNA modification pathways. RNA biology 2017, 14(9):1175-1184.

55. Thiaville JJ, Kellner SM, Yuan Y, Hutinet G, Thiaville PC, Jumpathong W, Mohapatra S, Brochier-Armanet C, Letarov AV, Hillebrand R: Novel genomic island modifies DNA with 7-deazaguanine derivatives. Proceedings of the National Academy of Sciences 2016, 113(11):E1452-E1459.

56. Smedley JG, Jewell E, Roguskie J, Horzempa J, Syboldt A, Stolz DB, Castric P: Influence of pilin glycosylation on Pseudomonas aeruginosa 1244 pilus function. Infection and immunity 2005, 73(12):7922-7931. 
57. Harvey H, Bondy-Denomy J, Marquis H, Sztanko KM, Davidson AR, Burrows LL: Pseudomonas aeruginosa defends against phages through type IV pilus glycosylation. Nature microbiology 2018, 3(1):47-52.

58. Vanaporn M, Titball RW: Trehalose and bacterial virulence. Virulence 2020, 11(1):1192-1202.

59. Collins J, Robinson C, Danhof H, Knetsch C, Van Leeuwen H, Lawley T, Auchtung J, Britton R: Dietary trehalose enhances virulence of epidemic Clostridium difficile. Nature 2018, 553(7688):291-294.

60. Schwarz S, Van Dijck P: Trehalose metabolism: a sweet spot for Burkholderia pseudomallei virulence. Virulence 2017, 8(1):5-7.

61. Mercier-Darty M, Royer G, Lamy B, Charron C, Lemenand O, Gomart C, Fourreau F, Madec J-Y, Jumas-Bilak E, Decousser J-W: Comparative Whole-Genome Phylogeny of Animal, Environmental, and Human Strains Confirms the Genogroup Organization and Diversity of the Stenotrophomonas maltophilia Complex. Applied and Environmental Microbiology 2020, 86(10).

62. Okazaki A, Avison MB: Induction of L1 and L2 $\beta$-lactamase production in Stenotrophomonas maltophilia is dependent on an AmpR-type regulator. Antimicrobial agents and chemotherapy 2008, 52(4):1525.

63. Mojica MF, Rutter JD, Taracila M, Abriata LA, Fouts DE, Papp-Wallace KM, Walsh TJ, LiPuma JJ, Vila AJ, Bonomo RA: Population structure, molecular epidemiology, and $\beta$ lactamase diversity among Stenotrophomonas maltophilia isolates in the United States. MBio 2019, 10(4).

64. Han L, Zhang R-M, Jia L, Bai S-C, Liu X-W, Wei R, Cui C-Y, Sun R-Y, Wang M-G, Cai D-T: Diversity of $L 1 / L 2$ genes and molecular epidemiology of high-level carbapenem resistance Stenotrophomonas maltophilia isolates from animal production environment in China. Infection, Genetics and Evolution 2020, 86:104531.

65. Barth E, Gora KV, Gebendorfer KM, Settele F, Jakob U, Winter J: Interplay of cellular cAMP levels, $\sigma S$ activity and oxidative stress resistance in Escherichia coli. Microbiology 2009, 155(Pt 5):1680.

66. Norton JP, Mulvey MA: Toxin-antitoxin systems are important for niche-specific colonization and stress resistance of uropathogenic Escherichia coli. PLOS Pathog 2012, 8(10):e1002954.

67. Miethke M, Klotz O, Linne U, May JJ, Beckering CL, Marahiel MA: Ferri-bacillibactin uptake and hydrolysis in Bacillus subtilis. Molecular microbiology 2006, 61(6):14131427.

68. Chouchane S, Girotto S, Kapetanaki S, Schelvis JP, Yu S, Magliozzo RS: Analysis of heme structural heterogeneity in Mycobacterium tuberculosis catalase-peroxidase (KatG). Journal of Biological Chemistry 2003, 278(10):8154-8162.

69. Kim Y, Maltseva N, Wilamowski M, Tesar C, Endres M, Joachimiak A: Structural and biochemical analysis of the metallo- $\beta$-lactamase $L 1$ from emerging pathogen Stenotrophomonas maltophilia revealed the subtle but distinct di-metal scaffold for catalytic activity. Protein Science 2020, 29(3):723-743.

70. Comolli JC, Hauser AR, Waite L, Whitchurch CB, Mattick JS, Engel JN: Pseudomonas aeruginosa gene products PilT and PilU are required for cytotoxicity in vitro and virulence in a mouse model of acute pneumonia. Infection and immunity 1999, 67(7):3625-3630.

71. Harvey KL, Jarocki VM, Charles IG, Djordjevic SP: The diverse functional roles of elongation factor Tu (EF-Tu) in microbial pathogenesis. Frontiers in microbiology 2019, 10:2351.

72. Pan H, Luan J, He X, Lux R, Shi W: The clpB gene is involved in the stress response of Myxococcus xanthus during vegetative growth and development. Microbiology 2012, 158(Pt 9):2336. 
73. Charoenlap N, Jiramonai L, Chittrakanwong J, Tunsakul N, Mongkolsuk S, Vattanaviboon P: Inactivation of ahpC renders Stenotrophomonas maltophilia resistant to the disinfectant hydrogen peroxide. Antonie Van Leeuwenhoek 2019, 112(5):809-814.

74. Chun H, Choi O, Goo E, Kim N, Kim H, Kang Y, Kim J, Moon JS, Hwang I: The quorum sensing-dependent gene katG of Burkholderia glumae is important for protection from visible light. Journal of bacteriology 2009, 191(13):4152-4157.

75. Li L-H, Shih Y-L, Huang J-Y, Wu C-J, Huang Y-W, Huang H-H, Tsai Y-C, Yang T-C: Protection from hydrogen peroxide stress relies mainly on AhpCF and KatA2 in Stenotrophomonas maltophilia. Journal of biomedical science 2020, 27(1):1-9.

76. Sánchez MB: Antibiotic resistance in the opportunistic pathogen Stenotrophomonas maltophilia. Frontiers in microbiology 2015, 6:658. 